\title{
A comparison of standard and high dose adenosine protocols in routine vasodilator stress cardiovascular magnetic resonance: dosage affects hyperaemic myocardial blood flow in patients with severe left ventricular systolic impairment
}

Louise A. E. Brown', Christopher E. D. Saunderson'1, Arka Das', Thomas Craven', Eylem Levelt', Kristopher D. Knott ${ }^{2,3}$, Erica Dall'Armellina', Hui Xue ${ }^{3}$, James C. Moon²,3, John P. Greenwood', Peter Kellman 1,2,3, Peter P. Swoboda ${ }^{1}$ and Sven Plein ${ }^{1 *}$ (D)

\begin{abstract}
Background: Adenosine stress perfusion cardiovascular magnetic resonance (CMR) is commonly used in the assessment of patients with suspected ischaemia. Accepted protocols recommend administration of adenosine at a dose of $140 \mu \mathrm{g} / \mathrm{kg} / \mathrm{min}$ increased up to $210 \mu \mathrm{g} / \mathrm{kg} / \mathrm{min}$ if required. Conventionally, adequate stress has been assessed using change in heart rate, however, recent studies have suggested that these peripheral measurements may not reflect hyperaemia and can be blunted, in particular, in patients with heart failure. This study looked to compare stress myocardial blood flow (MBF) and haemodynamic response with different dosing regimens of adenosine during stress perfusion CMR in patients and healthy controls.

Methods: 20 healthy adult subjects were recruited as controls to compare 3 adenosine perfusion protocols: standard dose $(140 \mu \mathrm{g} / \mathrm{kg} / \mathrm{min}$ for $4 \mathrm{~min})$, high dose $(210 \mu \mathrm{g} / \mathrm{kg} / \mathrm{min}$ for $4 \mathrm{~min}$ ) and long dose (140 $\mu \mathrm{g} / \mathrm{kg} / \mathrm{min}$ for $8 \mathrm{~min}) .60$ patients with either known or suspected coronary artery disease (CAD) or with heart failure and different degrees of left ventricular (LV) dysfunction underwent adenosine stress with standard and high dose adenosine within the same scan. All studies were carried out on a 3 T CMR scanner. Quantitative global myocardial perfusion and haemodynamic response were compared between doses.
\end{abstract}

Results: In healthy controls, no significant difference was seen in stress MBF between the 3 protocols. In patients with known or suspected CAD, and those with heart failure and mild systolic impairment (LV ejection fraction $(\mathrm{LVEF}) \geq 40 \%)$ no significant difference was seen in stress MBF between standard and high dose adenosine. In those

\footnotetext{
${ }^{*}$ Correspondence: s.plein@leeds.ac.uk

${ }^{1}$ Multidisciplinary Cardiovascular Research Centre (MCRC) \& Biomedical Imaging Science Department, Leeds Institute of Cardiovascular and Metabolic Medicine, University of Leeds, Clarendon Way, Leeds LS2 9JT, UK

Full list of author information is available at the end of the article
}

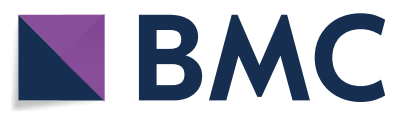

(c) The Author(s) 2021. Open Access This article is licensed under a Creative Commons Attribution 4.0 International License, which permits use, sharing, adaptation, distribution and reproduction in any medium or format, as long as you give appropriate credit to the original author(s) and the source, provide a link to the Creative Commons licence, and indicate if changes were made. The images or other third party material in this article are included in the article's Creative Commons licence, unless indicated otherwise in a credit line to the material. If material is not included in the article's Creative Commons licence and your intended use is not permitted by statutory regulation or exceeds the permitted use, you will need to obtain permission directly from the copyright holder. To view a copy of this licence, visit http://creativecommons.org/licenses/by/4.0/. The Creative Commons Public Domain Dedication waiver (http://creativeco mmons.org/publicdomain/zero/1.0/) applies to the data made available in this article, unless otherwise stated in a credit line to the data. 
with LVEF $<40 \%$, there was a significantly higher stress MBF following high dose adenosine compared to standard dose (1.33 \pm 0.46 vs $1.10 \pm 0.47 \mathrm{ml} / \mathrm{g} / \mathrm{min}, \mathrm{p}=0.004)$. Non-responders to standard dose adenosine (defined by an increase in heart rate $(\mathrm{HR})<10 \mathrm{bpm})$ had a significantly higher stress HR following high dose $(75 \pm 12 \mathrm{vs} 70 \pm 14 \mathrm{bpm}$, $p=0.034)$, but showed no significant difference in stress MBF.

Conclusions: Increasing adenosine dose from 140 to $210 \mu \mathrm{g} / \mathrm{kg} / \mathrm{min}$ leads to increased stress MBF in patients with significantly impaired LV systolic function. Adenosine dose in clinical perfusion assessment may need to be increased in these patients.

Keywords: Perfusion, Myocardial blood flow, Adenosine stress, Heart failure

\section{Introduction}

Stress perfusion cardiovascular magnetic resonance (CMR) is an accurate, non-invasive technique for the detection of myocardial ischaemia $[1,2]$. The method is widely used in the assessment of patients with suspected or known coronary artery disease (CAD); either to detect ischaemia, or in the context of cardiac dysfunction to detect an underlying ischaemic cause.

Intravenous adenosine has been shown to induce near maximal hyperaemia [3] and is used for assessment of ischaemia using both invasive measurements such as fractional flow reserve (FFR) and non-invasive techniques including CMR and positron emission tomography (PET). For stress perfusion CMR, adenosine is the most commonly used pharmacological stress agent [4]. Accepted protocols recommend administration of adenosine at a dose of $140 \mu \mathrm{g} / \mathrm{kg} / \mathrm{min}$ with an increase up to $210 \mu \mathrm{g} / \mathrm{kg} / \mathrm{min}$ if required to achieve adequate stress [5]. The duration of adenosine infusion is standardised and usually given for at least 3 min prior to data acquisition, but it is not known if a longer duration or higher dose may produce a better response.

Conventionally, adequate stress is defined by a heart rate (HR) rise of $\geq 10 \mathrm{bpm}$ or a systolic blood pressure (SBP) fall of $>10 \mathrm{mmHg}$ [5], based on the assumption that coronary vasodilatation leads to systemic vasodilation and reflex tachycardia. Recent studies have suggested however, that these peripheral measurements may not be a true reflection of myocardial hyperaemia and should not be used to assess adenosine response [6, 7]. In addition, certain patient groups including those with heart failure and diabetes mellitus have a blunted haemodynamic response to intravenous adenosine [8-10] and it is unclear to what extent this reduced response is reflected in coronary vasodilation.

Recently developed techniques of inline myocardial perfusion mapping with CMR provide accurate, reproducible assessment of rest and vasodilator stress myocardial blood flow (MBF) following adenosine administration [11-13]. This study looked to compare stress MBF with different dosing regimens of adenosine during stress perfusion CMR in patients with suspected CAD, heart failure and healthy controls.

\section{Methods}

\section{Study population}

Twenty healthy subjects were recruited as controls to compare 3 adenosine perfusion protocols. Exclusion criteria were any known cardiovascular disease, hypertension, hyperlipidaemia, diabetes mellitus, smoking, body mass index $(\mathrm{BMI})>30$ and any contraindication to CMR, adenosine or gadolinium-based contrast agents.

Sixty patients with symptoms of angina or heart failure were recruited prospectively from CMR or coronary angiography waiting lists, for comparison of 2 adenosine protocols determined from the results in healthy subjects. Patients were divided into three groups for analysis consisting of; Group 1-Patients with coronary artery disease and left ventricular (LV) ejection fraction (LVEF) $\geq 40 \%$. Group 2-Mild to moderate heart failure, LVEF $\geq 40 \%$ and no evidence of coronary disease, and Group 3-Moderate to severe heart failure, LVEF $<40 \%$ and no evidence of CAD. Exclusion criteria for patients were the presence of any contraindication to CMR, adenosine or gadolinium-based contrast agents. CAD was defined by either ischaemic (subendocardial) late gadolinium enhancement (LGE) on CMR, at least moderate stenosis on coronary angiography, previous percutaneous coronary intervention or previous coronary arterial bypass grafting.

\section{Study protocol}

All CMR studies were undertaken on a $3 \mathrm{~T}$ CMR system (Magnetom Prisma, Siemens Healthineers, Erlangen, Germany). Participants were advised to avoid caffeine for $24 \mathrm{~h}$ before the study. The protocol consisted of cine imaging, stress and rest perfusion, and LGE.

For perfusion imaging, adenosine was infused at a set dose for a preassigned time. Healthy subjects had three stress perfusion acquisitions; standard dose $(140 \mu \mathrm{g} / \mathrm{kg} /$ min adenosine for $4 \mathrm{~min})$, high dose $(210 \mu \mathrm{g} / \mathrm{kg} / \mathrm{min}$ for $4 \mathrm{~min}$ ) and long dose (140 $\mu \mathrm{g} / \mathrm{kg} / \mathrm{min}$ for $8 \mathrm{~min})$. Patients received standard dose and high dose for $4 \mathrm{~min}$ each. 


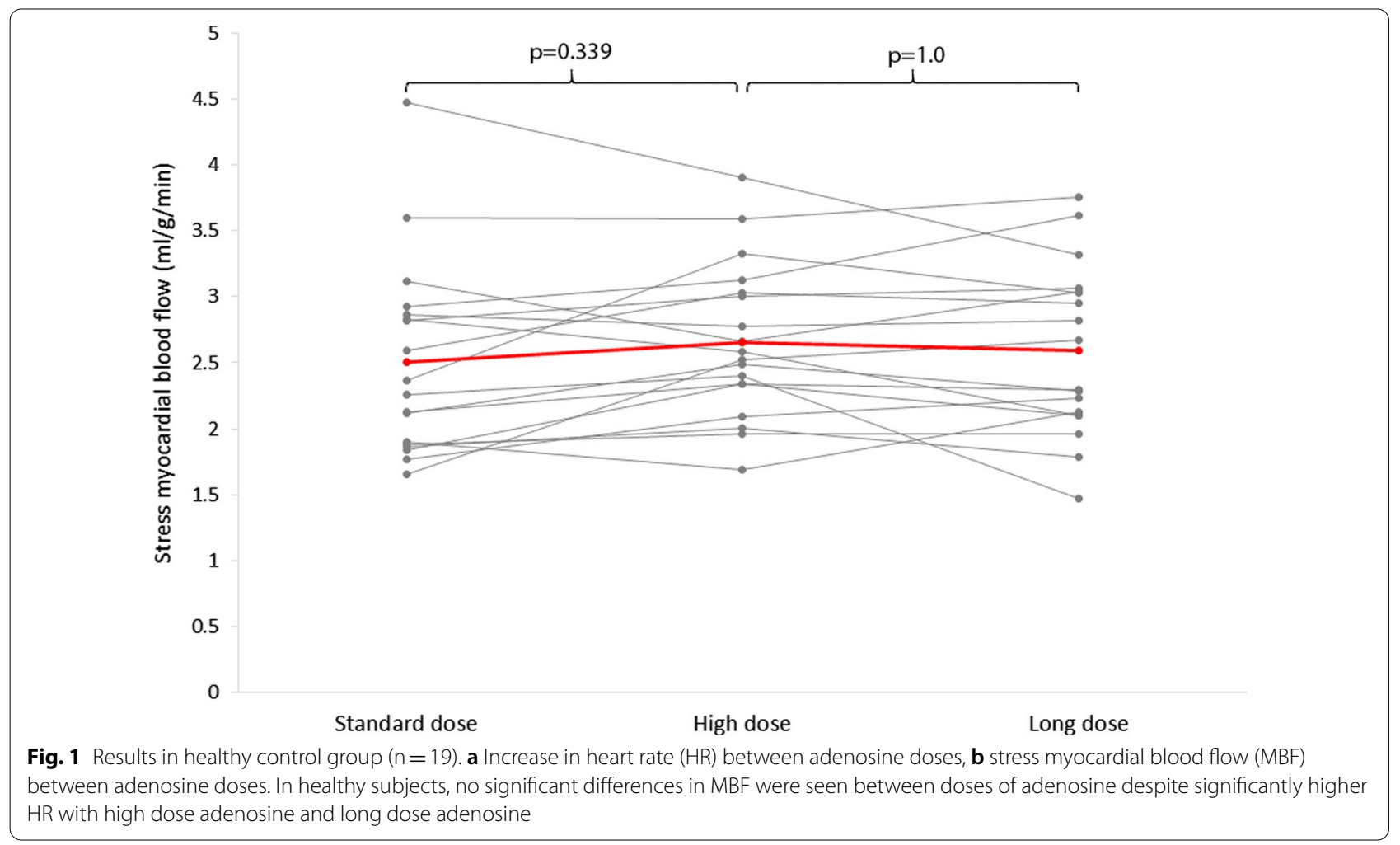

Doses were given in random order. A 10-min interval was kept between perfusion acquisitions, rest perfusion images were acquired $10 \mathrm{~min}$ after the final stress perfusion sequence in all participants.

Participants were monitored for symptoms throughout the scan. SBP and HR were recorded prior to starting adenosine infusion and before acquisition. For each perfusion acquisition, an intravenous bolus of $0.05 \mathrm{mmol} /$ kg gadobutrol (Gadovist, Bayer Healthcare, Berlin, Germany) was administered at $5 \mathrm{ml} / \mathrm{s}$ followed by a $20 \mathrm{ml}$ saline flush using an automated injection pump (Medrad MRXperion Injection System, Bayer Healthcare). Perfusion mapping was performed and implemented on the scanner using the Gadgetron streaming software image reconstruction framework as previously described [11, 13]. MBF maps were acquired as a short axis stack using a free-breathing, dual sequence, saturation recovery fast low angle shot (FLASH) protocol with motion correction. Three $8 \mathrm{~mm}$ slices were acquired, with slice spacing varied on a per patient basis to cover the left ventricle.

\section{Analysis}

Ischaemic segments were identified on visual assessment of perfusion images. Splenic switch off was assessed by comparing enhancement of splenic tissue at stress and at rest according to previously published methods $[14,15]$.
Perfusion maps were analysed using cvi42 software (Circle Cardiovascular Imaging, Calgary, Alberta, Canada). Endocardial and epicardial borders were drawn excluding papillary muscles, right ventricular insertion points marked, and a 16-segment American Heart Association model [16] used for further segmentation. In order to minimise partial volume effect, a $10 \%$ offset was applied to endocardial and epicardial borders [12]. MBF was recorded for each of the 16 segments. Where the LV outflow tract was included, or segments were too thin to contour, these segments were excluded from further analysis. Segments with ischaemic (subendocardial) LGE were also excluded from analysis. MBF values for all remaining segments were averaged to provide a value for global MBF.

\section{Subgroup analyses}

Subgroup analysis was carried out comparing patients with a HR change of $<10 \mathrm{bpm}$ (non-responders) compared to those with a rise of $\geq 10 \mathrm{bpm}$ (responders). Within Group 1, segments with ischaemia on visual assessment were compared with non-ischaemic segments. 


\section{Statistical analysis}

Analysis was performed using SPSS (version 23, Statistical Package for the Social Sciences, International Business Machines, Inc., Armonk, New York, USA). Normality of distribution was assessed using ShapiroWilk test. Different dosing regimens were analysed using paired t-tests or Wilcoxson Signed Rank test in patients and analysis of variance (ANOVA) with repeated measures and post-hoc Bonferroni correction, or the Kruskal Wallis $\mathrm{H}$ test in healthy controls. Categorical data was analysed using chi-square test or Fisher's exact test when expected numbers were $<5$. All statistical tests were twotailed and $\mathrm{p}<0.05$ was considered significant.

Myocardial perfusion reserve (MPR) was calculated as stress MBF:rest MBF. Inadequate HR response was defined as $<10 \mathrm{bpm}$ in keeping with Society for Cardiovascular Magnetic Resonance guidelines [5].

\section{Results}

\section{Healthy controls}

20 healthy control subjects were recruited $(25 \pm 2.7$ years, LVEF $57 \pm 3.3 \%)$. One subject withdrew after the first dose of adenosine. Haemodynamic data were available for all three dosing regimens for 19 healthy subjects. In one case artefact on perfusion maps at standard dose meant these were not included in analysis of MBF response. The final cohort therefore consisted of 18 subjects.

\section{Haemodynamic response}

Haemodynamic data are shown in Table 1. For all doses there was a significant rise in HR at stress from rest $(\mathrm{p}<0.001)$. In both standard and long dose protocols there was a significant increase in stress SBP from rest $(\mathrm{p}<0.01)$. There was no significant change in SBP between rest and high dose adenosine. Only one participant had a SBP decrease of $\geq 10 \mathrm{mmHg}$ at standard and high dose adenosine, and none had this degree of change with long dose.

There was a significant difference in stress HR and between standard and high dose adenosine, but no difference between standard and long dose. No significant difference was seen in SBP or change in SBP between doses.

\section{MBF}

Global stress MBF was $2.50 \pm 0.74 \mathrm{ml} / \mathrm{g} / \mathrm{min}$ with standard dose adenosine, with values of $2.66 \pm 0.59 \mathrm{ml} / \mathrm{g} / \mathrm{min}$ after high dose and $2.59 \pm 0.64 \mathrm{ml} / \mathrm{g} / \mathrm{min}$ with long dose, overall there was no significant difference between the three groups $(\mathrm{p}=0.32)$.

Although there was no significant difference in MBF, the largest increases in HR were seen following the high

Table 2 Characteristics of patient groups

\begin{tabular}{lllll}
\hline & Group 1 & Group 2 & Group 3 & $p$ \\
\hline $\mathrm{n}$ & 20 & 16 & 24 & \\
Sex-male & $17(85 \%)$ & $8(50 \%)$ & $17(71 \%)$ & 0.074 \\
Age & $62.9 \pm 8.7$ & $63.5 \pm 16.3$ & $65.1 \pm 12.7$ & 0.344 \\
Diabetes mellitus & $4(20 \%)$ & 0 & $6(25 \%)$ & 0.102 \\
Beta blocker usage & $14(70 \%)$ & $12(75 \%)$ & $17(71 \%)$ & 0.940 \\
LVEF & $57.5 \pm 7.9$ & $48.1 \pm 5.2$ & $26.2 \pm 7.0$ & $<0.001$ \\
\hline
\end{tabular}

Other than left ventricular ejection fraction (LVEF), no significant difference was seen between the groups in factors previously reported to affect adenosine response

Data given as mean \pm standard deviation or $\mathrm{n}(\%)$

Group 1-Patients with coronary disease and left ventricular ejection fraction (LVEF) $>40 \%$. Group 2-Mild to moderate heart failure, LVEF $\geq 40 \%$ and no evidence of coronary artery disease. Group 3-moderate to severe heart failure, LVEF $<40 \%$ and no evidence of coronary artery disease

Table 1 Haemodynamic response and MBF in healthy subjects

\begin{tabular}{|c|c|c|c|c|}
\hline & \multicolumn{3}{|l|}{ Adenosine dosing } & \multirow[b]{2}{*}{$p$} \\
\hline & $\begin{array}{l}\text { Standard dose: } 140 \mu \mathrm{g} / \mathrm{kg} / \mathrm{min} \\
4 \text { min duration }\end{array}$ & $\begin{array}{l}\text { High dose: } 210 \mu \mathrm{g} / \mathrm{kg} / \mathrm{min} \\
4 \text { min duration }\end{array}$ & $\begin{array}{l}\text { Long dose: } 140 \mu \mathrm{g} / \mathrm{kg} / \mathrm{min} \\
8 \text { min duration }\end{array}$ & \\
\hline Rest HR (bpm) & $71 \pm 15$ & $71 \pm 14$ & $71 \pm 13$ & 0.962 \\
\hline Rest SBP (mmHg) & $117 \pm 12$ & $118 \pm 14$ & $117 \pm 13$ & 0.481 \\
\hline Stress HR (bpm) & $102 \pm 19^{\dagger}$ & $108 \pm 16^{\dagger}$ & $106 \pm 19^{\dagger}$ & $0.017^{*}$ \\
\hline Stress SBP (mmHg) & $121 \pm 16^{\dagger}$ & $121 \pm 16$ & $124 \pm 17^{\ddagger}$ & 0.195 \\
\hline Change in HR (bpm) & $30 \pm 12$ & $37 \pm 11$ & $35 \pm 13$ & $0.045^{*}$ \\
\hline Change in SBP (mmHg) & $5 \pm 6$ & $3 \pm 9$ & $7 \pm 11$ & 0.072 \\
\hline Stress MBF (ml/g/min) & $2.50 \pm 0.74$ & $2.66 \pm 0.59$ & $2.59 \pm 0.64$ & 0.323 \\
\hline MPR & $3.52 \pm 0.93$ & $3.82 \pm 0.83$ & $3.72 \pm 1.00$ & 0.191 \\
\hline
\end{tabular}

A significant difference was seen in stress heart rate and change in stress $\mathrm{HR}$

$H R$ heart rate, SBP systolic blood pressure, MBF myocardial blood flow, MPR myocardial perfusion reserve

${ }^{*}$ Significant difference between adenosine doses, ${ }^{\dagger}$ significant difference from rest value, $p<0.05^{\ddagger}$ significant difference from rest value, $p<0.01$. Data given as mean \pm standard deviation 
dose regimen (Fig. 1), therefore this regime was chosen for comparison with standard dose in patients.

\section{Patients}

Sixty patients took part in the study divided into 20 in Group 1 (CAD), 16 in Group 2 (mild to moderate heart failure, $\mathrm{LVEF} \geq 40 \%$ ) and 24 in Group 3 (moderate to severe heart failure, $\mathrm{LVEF}<40 \%$ ). No significant differences were seen between the groups in incidence of diabetes mellitus, beta-blocker usage, age or sex (Table 2).

\section{Haemodynamic response}

Haemodynamic data are shown in Table 3. Mean stress HR increased significantly from mean rest $H R$ in all groups and following both standard and high dose adenosine $(\mathrm{p}<0.01)$. There was no significant change between stress and rest SBP in any group. In total, 36 (60\%) patients had a HR rise $\geq 10 \mathrm{bpm}$ with standard dose adenosine, and 42 (70\%) with high dose. One (2\%) patient had a SBP decrease of $\geq 10 \mathrm{mmHg}$ with standard dose, and 2 (3\%) with high dose. In each group, there was a similar proportion of non-responders.

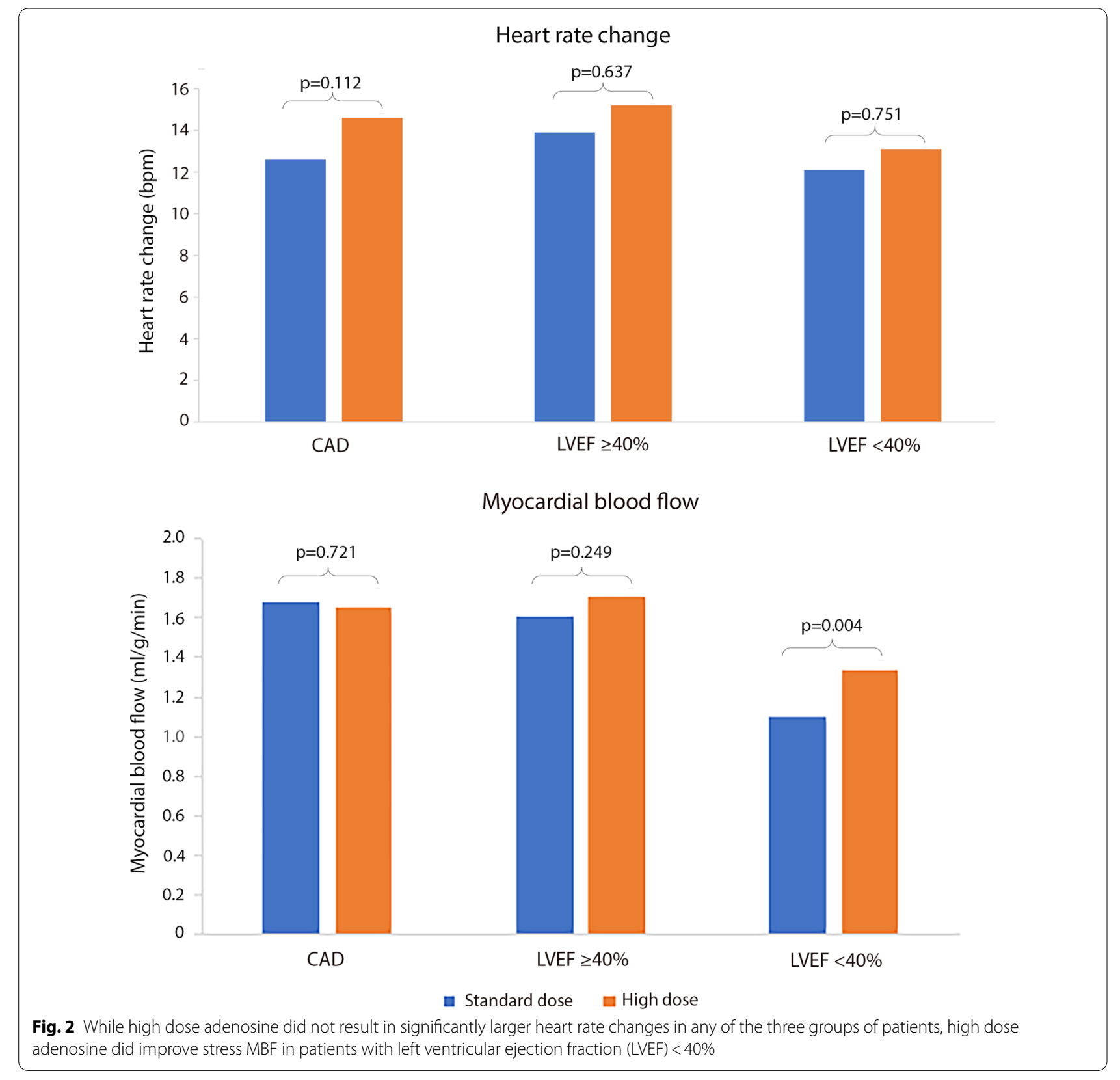




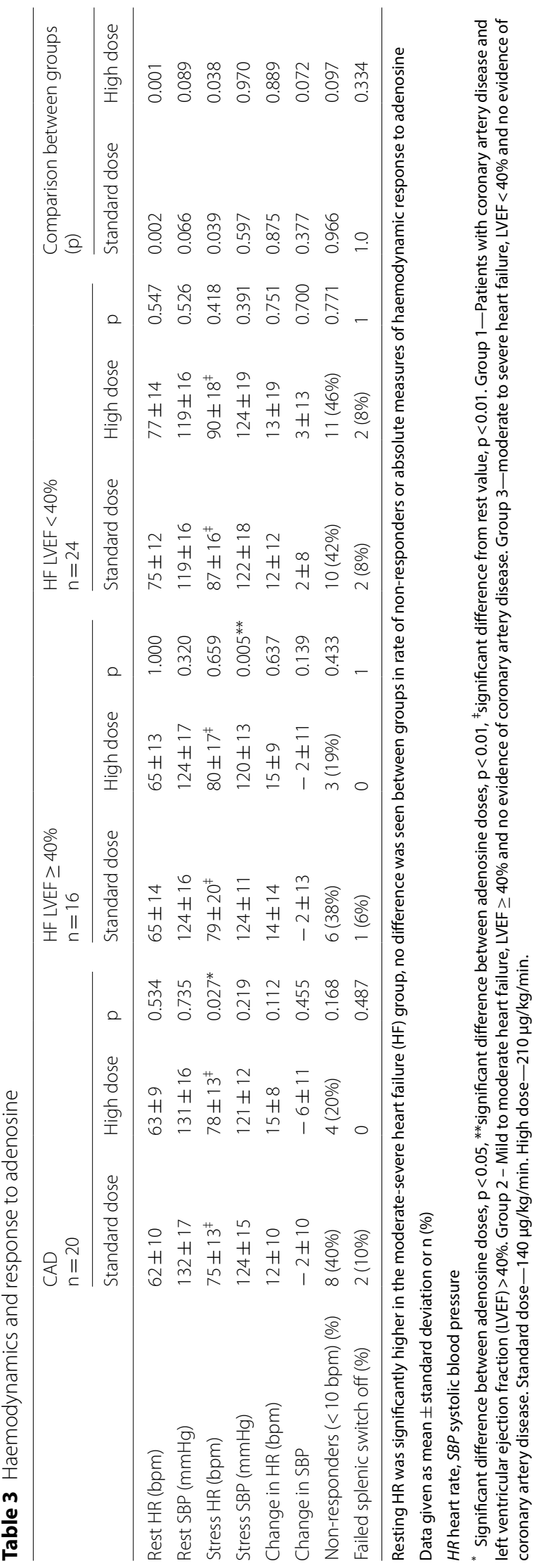


Within group 1, stress HR was significantly higher following high dose compared with standard dose adenosine (78 \pm 13 vs $75 \pm 13 \mathrm{bpm}, \mathrm{p}=0.025$ ), but no significant difference was seen in groups 2 or 3 . Stress SBP was significantly lower following high dose adenosine compared to standard dose in group $2(120 \pm 13$ vs $124 \pm 11 \mathrm{mmHg}$, $\mathrm{p}=0.005)$. There was no significant difference in stress SBP between doses in the other groups.

No significant difference was seen in absolute HR rise between the groups (Fig. 2) and no significant correlation was seen between LVEF and HR rise across all 60 patients $(\mathrm{r}=0.122, \mathrm{p}=0.353)$.

\section{MBF and MPR}

No difference was seen in rest MBF between the 3 groups (Group $1-0.73 \pm 0.16 \mathrm{ml} / \mathrm{g} / \mathrm{min}$, Group $2-0.71 \pm 0.23 \mathrm{ml} / \mathrm{g} / \mathrm{min}$, Group $3-0.62 \pm 0.16 \mathrm{ml} / \mathrm{g} /$ min, $\mathrm{p}=0.144)$. No difference was seen in MBF or MPR between different adenosine doses in either Group 1 or 2. Within Group 3, MBF was significantly higher following high dose than after standard dose adenosine (standard dose $1.10 \pm 0.47$ vs high dose $1.33 \pm 0.46 \mu \mathrm{g} / \mathrm{ml} / \mathrm{min}$, $\mathrm{p}=0.004$ ) (Figs. 2, 3). MPR demonstrated the same pattern (standard dose $1.90 \pm 0.88$ vs high dose $2.26 \pm 0.90$, $\mathrm{p}=0.004)$.

Bland Altman plots (Fig. 4) show the spread of differences in MBF between adenosine doses and those with and without adequate response to adenosine at standard dose.

There were weak correlations between increase in HR and MPR both with standard $(\mathrm{r}=0.266, \mathrm{p}=0.045)$ and high dose adenosine $(\mathrm{r}=0.54, \mathrm{p}<0.001)$. HR response with standard dose adenosine did not correlate with an increase in MBF or MPR with high dose adenosine.

Five patients demonstrated failed splenic switch off with standard dose adenosine (Table 3). Analysis was repeated, excluding those with failed splenic switch off. These showed the same pattern with no difference in stress MBF or MPR between standard and high dose adenosine in Group 1 and Group 2, but higher stress MBF $(1.15 \pm 0.46$ vs $1.38 \pm 0.45, \mathrm{p}=0.009)$ and $\mathrm{MPR}$ $(1.99 \pm 0.88$ vs $2.34 \pm 0.91, \mathrm{p}=0.009)$ in Group 3 .

\section{Subgroup analyses}

\section{Heart rate response to adenosine}

Patients were divided into groups of non-responders $(\mathrm{n}=24)$ and responders $(\mathrm{n}=36)$ based on a HR increase of $<10 \mathrm{bpm}$ or $\geq 10 \mathrm{bpm}$. There was no significant difference in LVEF, age or incidence of diabetes or beta blocker usage between the two groups. Stress HR was significantly different between groups both with standard and high dose adenosine $(70 \pm 14$ vs $88 \pm 15 \mathrm{bpm}, \mathrm{p}=<0.001$ at standard dose and $75 \pm 12$ vs $89 \pm 18 \mathrm{bpm}, \mathrm{p}=0.001$ at high dose). There was no significant difference in rest HR or stress MBF between the two groups at either adenosine dose.

In the non-responder group, stress HR was significantly higher with high dose adenosine than with standard dose (Table 4). Those with adequate HR response to standard dose did not have a significant increase in stress HR between doses. No significant difference was seen in stress MBF between standard and high dose adenosine regardless of adequate $\mathrm{HR}$ response to standard dose adenosine.

\section{Ischaemia}

Within group 1, 11 patients had evidence of regional inducible ischaemia. On visual analysis, ischaemia was seen in the same coronary territories between the adenosine doses. A total of 60 ischaemic segments were visually identified following standard dose adenosine, and 63 segments following high dose. No significant difference was seen in MBF in between standard and high dose adenosine in either the ischaemic $(1.46 \pm 0.30$ vs $1.48 \pm 0.40 \mathrm{ml} / \mathrm{g} / \mathrm{min}, \mathrm{p}=0.697)$ or non-ischaemic segments $(1.72 \pm 0.57$ vs $1.77 \pm 0.52 \mathrm{ml} / \mathrm{g} / \mathrm{min}, \mathrm{p}=0.130)$ in these patients.

\section{Discussion}

The Society for Cardiovascular Magnetic Resonance Standardized (SCMR) imaging protocols for stress perfusion recommend an adenosine dose of $140 \mu \mathrm{g} / \mathrm{kg}$ body weight/min for 2-4 min with an increase in the dose if there is inadequate HR and SBP response [5]. Our results inform several aspects of this recommendation: the duration of adenosine infusion, the dose of adenosine and the use of HR and SBP as indicators of adequate response. In healthy controls, increased dose or extended duration adenosine were not associated with significant changes in stress MBF compared with standard dose adenosine. Equally, in patients with normal or mildly impaired LV systolic function there was no effect of higher dose adenosine, but in those with moderate to severe LV systolic dysfunction (LVEF $\leq 40 \%$ ), higher dose adenosine produced higher stress MBF. We further show that HR and SBP are unreliable markers of haemodynamic response.

\section{Duration of adenosine infusion}

The duration of adenosine infusion has not previously been studied for stress perfusion CMR but has been the subject of studies in nuclear cardiology. In Single Photon Emission Computed Tomography, a 3-min adenosine infusion showed better tolerability with similar diagnostic performance compared with a 6-min protocol[17]. A PET study using Rb-82 compared several adenosine regimes in 127 subjects and found that a 6-min adenosine 


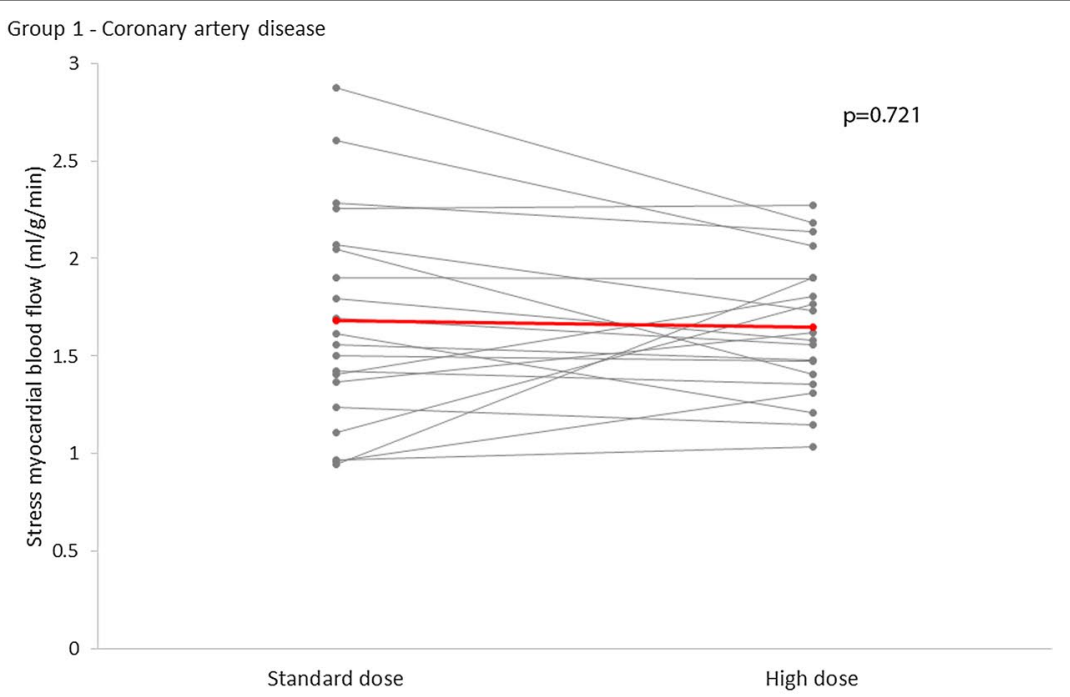

Group 2 - Heart Failure - $E F \geq 40 \%$

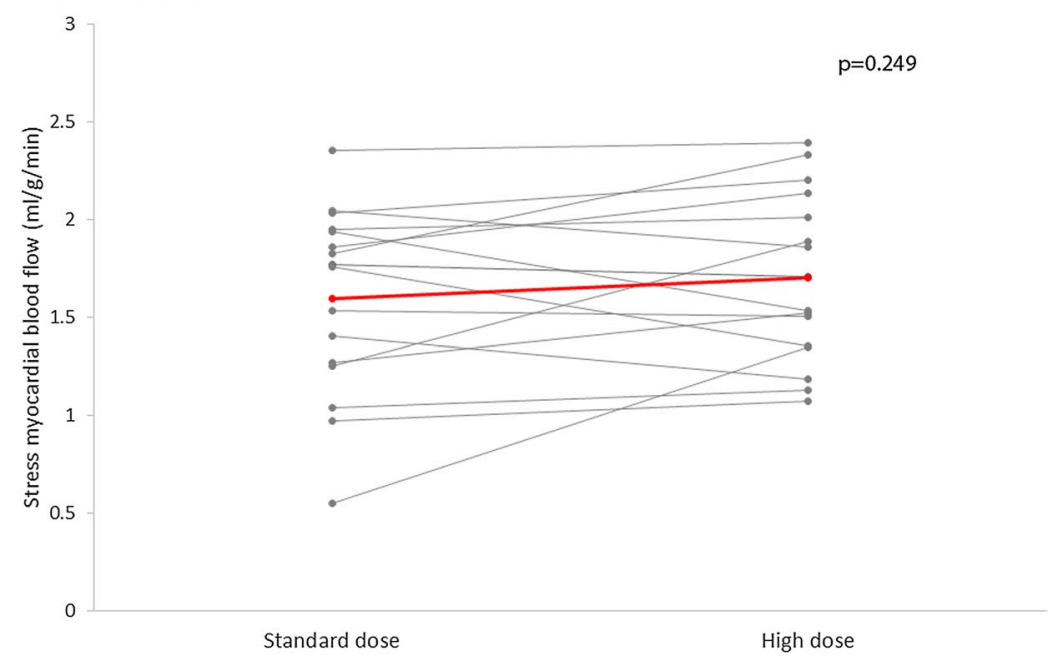

Group 3 - Heart Failure - EF $<40 \%$

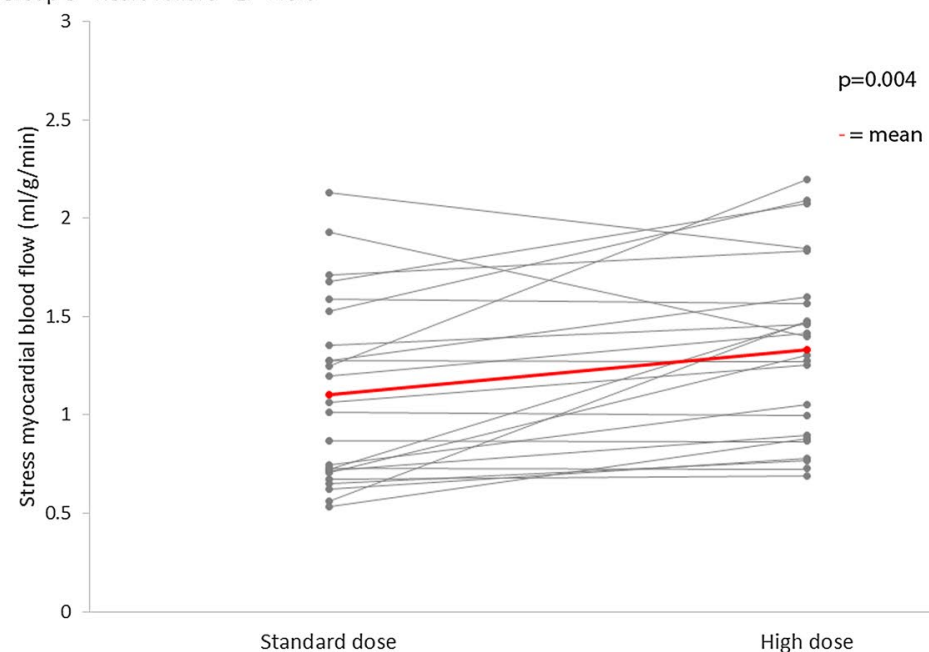

Fig. 3 Difference in stress MBF between doses in three groups of patients. Within Group 3 (moderate-severe heart failure) stress MBF was significantly higher with high dose adenosine compared to standard dose 

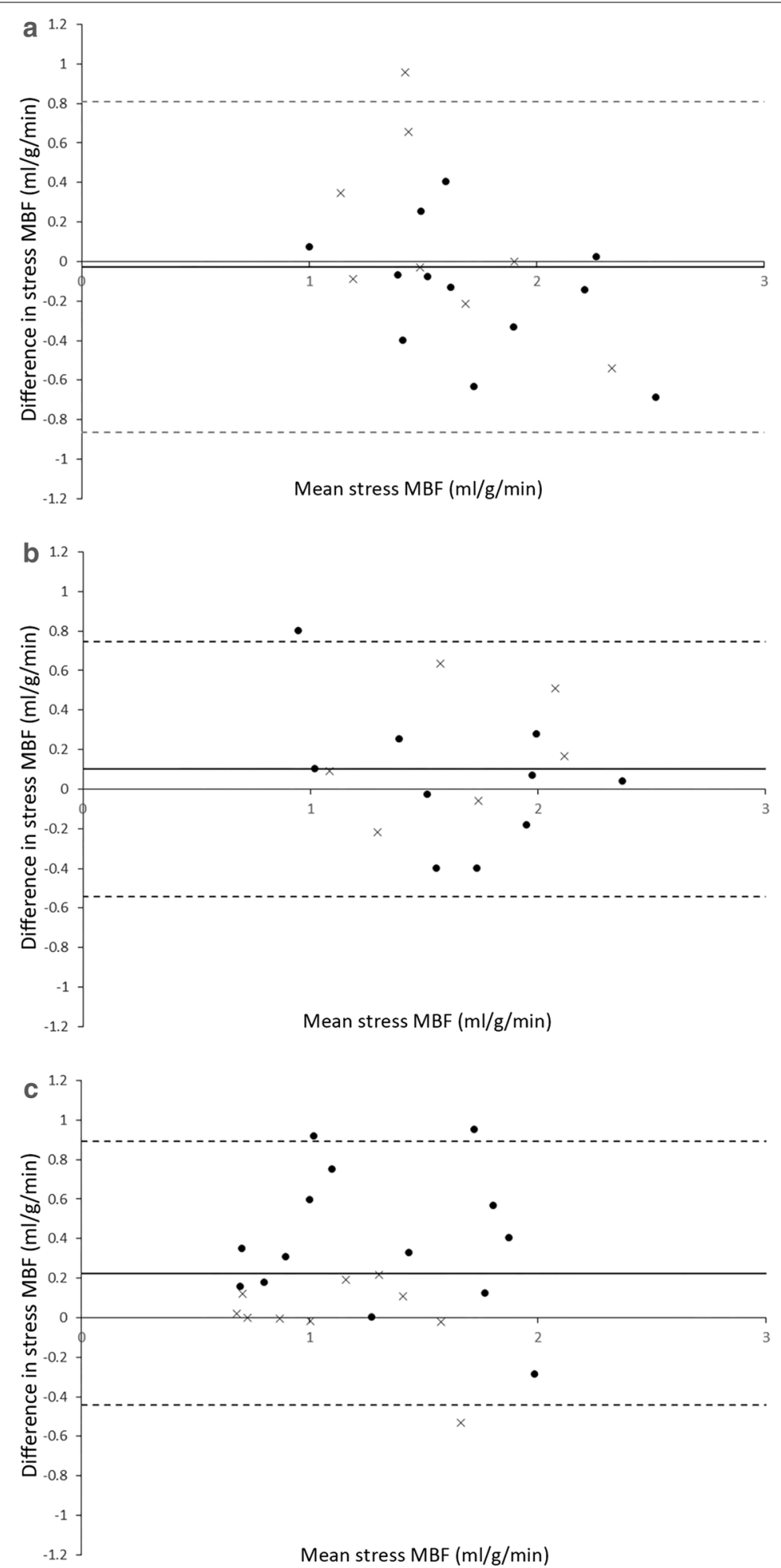

$-210 \mathrm{bpm}$ rise in HRwith standard dose $x<10 \mathrm{bpm}$ rise in $\mathrm{HR}$ with standard dose

Fig. 4 Difference in MBF between doses. represents adequate HR response to standard dose adenosine, $x$ represents blunted response. In Group 3 , stress MBF is significantly higher following high dose adenosine, this effect does not appear to be related to HR response at standard dose 
Table 4 Difference in response to adenosine doses divided by $\mathrm{HR}$ response to standard dose

\begin{tabular}{lccc}
\hline & Standard dose & High dose & $p$ \\
\hline Non-responders $(\mathrm{n}=24)$ & & & \\
Rest HR $(\mathrm{bpm})$ & $67 \pm 16$ & $68 \pm 14$ & 0.789 \\
Stress HR (bpm) & $70 \pm 14$ & $75 \pm 12$ & 0.034 \\
Increase in HR (bpm) & $3 \pm 6$ & $7 \pm 11$ & 0.053 \\
Stress MBF (ml/g/min) & $1.35 \pm 0.49$ & $1.45 \pm 0.46$ & 0.188 \\
MPR & $1.93 \pm 0.77$ & $2.05 \pm 0.70$ & 0.215 \\
Adequate HR response $(\mathrm{n}=36)$ & & \\
Rest HR (bpm) & $69 \pm 12$ & $70 \pm 13$ & 0.461 \\
Stress HR (bpm) & $88 \pm 15$ & $89 \pm 18$ & 0.762 \\
Increase in HR (bpm) & $19 \pm 9$ & $19 \pm 13$ & 0.838 \\
Stress MBF $(\mathrm{ml} / \mathrm{g} / \mathrm{min})$ & $1.48 \pm 0.60$ & $1.59 \pm 0.43$ & 0.103 \\
MPR & $2.32 \pm 0.86$ & $2.53 \pm 0.63$ & 0.073 \\
\hline
\end{tabular}

Stress HR was significantly higher with high dose adenosine in the nonresponder group only. Non-responders and responders were defined by heart rate increase with adenosine of $<10 \mathrm{bpm}$ or $\geq 10 \mathrm{bpm}$. There data are from patients only. No difference was seen in stress MBF in either group

Data given as mean \pm standard deviation or $\mathrm{n}(\%)$

$H R$ heart rate, $M B F$ myocardial blood flow

infusion protocol with $\mathrm{Rb}-82$ activation at 3 min was associated with $11.4 \%$ higher stress MBF and $15.7 \%$ higher coronary flow reserve (CFR) than a 4-min adenosine infusion with Rb-82 activation at 2 min [18]. Further extension of the adenosine infusion time prior to $\mathrm{Rb}-82$ activation did not increase increased stress MBF or CFR further. These results are not directly applicable to CMR due to the differences in tracer kinetics and data capture between PET and CMR. Our data show that in myocardial perfusion CMR, there is no significant difference in haemodynamic response and no change in quantitative MBF between a $4 \mathrm{~min}$ and an extended $8 \mathrm{~min}$ adenosine protocol in healthy subjects. These results suggest that the shorter duration protocols that are in current clinical use and recommended in current guidance are adequate for myocardial perfusion CMR.

\section{Dose of adenosine infusion}

The dose of adenosine infusion has been studied more extensively, using multiple modalities. Early invasive studies using intracoronary Doppler assessment of coronary blood flow velocity and total coronary resistance showed that intravenous adenosine at doses of $140 \mu \mathrm{g} /$ $\mathrm{kg} / \mathrm{min}$ resulted in maximal hyperaemia, defined by papaverine response, in $84 \%$ of subjects [3]. Several invasive studies have assessed the effect of adenosine dose on FFR with higher doses showing no significant change in FFR compared with lower doses [19-21]. In a CMR study, Karamitsos et al. showed that a stepwise increase in the adenosine dose from $140 \mu \mathrm{g} / \mathrm{kg} / \mathrm{min}$ to $210 \mu \mathrm{g} / \mathrm{kg} /$ min is safe and increases the rate of patients with an adequate haemodynamic response [22]. However, the MBF response to different adenosine doses has not previously compared using quantitative myocardial perfusion CMR. In addition to studying the same individuals repeatedly, we obtained MBF values at different adenosine doses in the same imaging session. This approach overcomes the potential confounders of day-to-day physiological variation in haemodynamic response and allows direct comparison of dose effects. Our data show no significant difference in MBF following standard and high dose adenosine in healthy subjects and patients with CAD or heart failure with $\mathrm{LVEF} \geq 40 \%$, suggesting that irrespective of haemodynamic response, standard dose adenosine in these groups reliably induces maximal hyperaemia.

\section{Impaired LV systolic function}

In patients with severe systolic impairment, previous studies have shown a blunted HR response to adenosine, with an increase in adenosine from 140 to $210 \mu \mathrm{g} /$ $\mathrm{kg} / \mathrm{min}$ more commonly required to achieve a sufficient haemodynamic response [8]. In CMR, a previous study reported $\mathrm{LVEF}<57 \%$ as an independent predictor of inadequate haemodynamic response to standard adenosine dose [22]. Within our patient cohort, there was no correlation between LVEF and HR rise, and no difference in HR rise between the patient groups, or any significant difference in the rate of non-responders. However, our study showed for the first time that among patients with heart failure and significant LV systolic impairment (LVEF $\leq 40 \%)$, stress MBF increases with higher doses of adenosine, suggesting that standard dose regimes fail to induce maximal hyperaemia and are not appropriate in these patients. A pattern of decreased response to adenosine in heart failure requiring higher doses to achieve stress has previously been suggested [8]. Potential mechanisms for the lower adenosine effect include the downregulation of gene expression of both adenosine receptors and adenosine deaminase in impaired myocardium, together with a decrease in the activity of adenosine deaminase [23, 24]. Increased levels of cardiac adenosine have also been measured in chronic heart failure patients, and this higher endogenous level may explain the requirement for higher exogenous doses to achieve the anticipated vasodilation required in stress testing $[23,24]$.

\section{Haemodynamic response}

In non-invasive testing, response to adenosine and the achievement of hyperaemia is commonly assessed using haemodynamic response relating to peripheral vasodilation. Conventionally, an increase in $\mathrm{HR}$ by $>10 \mathrm{bpm}$ and a fall in systolic SBP by $>10 \mathrm{mmHg}$ are considered markers 
of adequate hyperaemia [5]. A small previous PET perfusion study suggested a correlation between HR response and stress MBF [18], but confounders such as LVEF were not explicitly considered. In an earlier larger PET study, change in HR correlated poorly with stress MBF, and not with CFR, leading the authors to suggest that peripheral haemodynamic changes could not be used to assess the adequacy of response to adenosine [6]. No CMR studies have previously looked at haemodynamic response and change in quantitated MBF.

Within our study, $61 \%$ of those patients with $\mathrm{LVEF}>40 \%$ reached the threshold of $10 \mathrm{bpm}$, comparable to published results in other studies with a similar patient group [8]. This relatively low response rate may be due to the presence of medications and other co-morbidities in our patient cohort. Blunted haemodynamic response to adenosine has been reported in diabetes, beta-blocker usage and CAD as well as impaired LVEF [8-10, 22, 25]. Increase in HR correlated only weakly with MPR and no significant difference was seen in stress MBF between groups classified by HR response.

This study showed no significant relationship between rise in HR at standard dose adenosine and an increase in stress MBF or MPR with high dose adenosine. This indicates that in patients with a low HR response at standard dose adenosine, a higher dose does not increase myocardial perfusion-a finding that questions the validity of current guideline recommendations and widely used clinical practice.

Further, we saw no significant difference in SBP change between groups of patients, or adenosine doses and $<3 \%$ of patients had a decrease in SBP of $\geq 10 \mathrm{mmHg}$ as described in standard protocols. SBP even increased in healthy subjects over baseline. These data suggest, in keeping with previous studies, that in particular SBP response should not be used as a marker of adequate vasodilator response, possibly due to an adrenergic response to adenosine symptoms, which overcomes the vasodilator effects on blood pressure.

\section{Clinical implications}

Our data suggest that those with reduced LVEF should have higher dose adenosine to achieve maximal hyperaemia, regardless of haemodynamic response. Our data also question the use of HR and SBP response to standard dose adenosine as criteria to increase the adenosine dose as it does not appear to increase MBF. However, due to low numbers of patients with inducible ischaemia in this study we have not been able to assess the diagnostic impact of our observations although we observed a small, non-significant, increase in the number of ischaemic segments identified following high dose adenosine.

\section{Limitations}

Our data may be influenced by physiological variation, although we have tried to minimise this. It is possible some effects may not have been controlled for, although we have previously demonstrated no significant difference in serial measurements of stress MBF within a CMR study [12]. Caffeine has been demonstrated to affect adenosine stress perfusion CMR [26]. Although we advised our subjects to avoid caffeine for $24 \mathrm{~h}$ prior to the scan, previous studies have demonstrated that up to $20 \%$ may still have detectable caffeine levels [27] and we cannot account for how these may be distributed between our patient groups in this study. The age range of our healthy subjects was considerably lower than those of the patient groups, if age influences the response to adenosine then the results from these healthy subjects may not be applied to our patient groups. There was no significant difference in mean ages between the patient groups. Our data cannot exclude that a higher dose of adenosine than $210 \mu \mathrm{g} / \mathrm{kg} / \mathrm{min}$ might further increase stress MBF in those with $\operatorname{LVEF}<40 \%$, but our data also cannot be extrapolated to support this possibility. Although the use of doses in excess of $210 \mathrm{mcg} / \mathrm{kg} / \mathrm{min}$ are not used in routine practice, future studies should explore higher doses in particular in HF patients.

\section{Conclusions}

Increasing adenosine dose is well tolerated and related to increased stress myocardial blood flow in patients with significant LV impairment. Achievement of adequate myocardial vasodilator response, assessed by quantitative perfusion, is not significantly related to peripheral haemodynamic measurements SBP and HR. These observations may impact future practice guidelines for stress perfusion CMR. Dosage of adenosine in clinical perfusion assessment should be carefully considered and may need to be increased in subsets of patients, in particular those with severely impaired LV function, or alternative stress agents considered.

\section{Abbreviations}

BMI: Body mass index; CAD: Coronary artery disease; CFR: Coronary flow reserve; CMR: Cardiovascular magnetic resonance; FFR: Fractional flow reserve; HF: Heart failure; HR: Heart rate; LGE: Late gadolinium enhancement; LV: Left ventricle/left ventricular; LVEF: Left ventricular ejection fraction; MBF: Myocardial blood flow; MPR: Myocardial perfusion reserve; PET: Positron emission tomography; SBP: Systolic blood pressure.

\section{Acknowledgements}

We thank the CMR team at Leeds General Infirmary for their help in recruiting and scanning patients and the organisation of running this study. Dr. Andrew Arai served as a JCMR Guest Editor for this manuscript. 


\section{Authors' contributions}

LB: Conception and design, recruitment, scanning and supervision of scans, analysis and interpretation of data, drafting of manuscript. CS: recruitment, supervision of scans, critical and intellectual revision of manuscript. AD: critical and intellectual revision of manuscript. AD: critical and intellectual revision of manuscript TC: critical and intellectual revision of manuscript EL: critical and intellectual revision of manuscript KK: critical and intellectual revision of manuscript ED: critical and intellectual revision of manuscript $H X$ : processing and interpretation of CMR data, critical and intellectual revision of manuscript JM: critical and intellectual revision of manuscript JG: critical and intellectual revision of manuscript PK: processing and interpretation of CMR data, critical and intellectual revision of manuscript PS: recruitment, supervision of scans, critical and intellectual revision of manuscript, SP: concept and design and drafting of manuscript. All authors read and approved the final manuscript.

\section{Funding}

This work was supported by British Heart Foundation grants $\mathrm{CH} / 16 / 2 / 32089$ and $R G / 16 / 1 / 32092$ to Prof Sven Plein.

\section{Availability of data and materials}

The datasets used and/or analysed during the current study are available from the corresponding author on reasonable request.

\section{Ethics approval and consent to participate}

The study was performed in accordance with the Declaration of Helsinki (October 2000), with all participants providing informed written consent. Ethical approval was given by NRES Committee Yorkshire and the Humber-Leeds West, Ref 12/YH/0551 and Ref 17/YH/0300.

\section{Consent for publication}

Not applicable.

\section{Competing interests}

The authors declare that they have no competing interests.

\begin{abstract}
Author details
${ }^{1}$ Multidisciplinary Cardiovascular Research Centre (MCRC) \& Biomedical Imaging Science Department, Leeds Institute of Cardiovascular and Metabolic Medicine, University of Leeds, Clarendon Way, Leeds LS2 9JT, UK. ${ }^{2}$ The Cardiovascular Magnetic Resonance Imaging Unit and The Inherited Cardiovascular Diseases Unit, Barts Heart Centre, St Bartholomew's Hospital, West Smithfield, London, UK. ${ }^{3}$ National Heart, Lung, and Blood Institute, National Institutes of Health, DHHS, Bethesda, MD, USA.
\end{abstract}

Received: 27 November 2019 Accepted: 26 January 2021

Published online: 18 March 2021

\section{References}

1. Greenwood JP, Maredia N, Younger JF, Brown JM, Nixon J, Everett CC, et al. Cardiovascular magnetic resonance and single-photon emission computed tomography for diagnosis of coronary heart disease (CE-MARC): a prospective trial. Lancet. 2012;379(379):453-60.

2. Greenwood JP, Ripley DP, Berry C, McCann GP, Plein S, Bucciarelli-Ducci C et al. Effect of care guided by cardiovascular magnetic resonance, myocardial perfusion scintigraphy, or NICE guidelines on subsequent unnecessary angiography rates : the CE-MARC 2 randomized clinical trial. JAMA. 2016;316(10):1051-60.

3. Wilson RF, Wyche K, Christensen BV, Zimmer S, Laxson DD. Clinical investigation effects of adenosine on human coronary arterial circulation. Circulation. 1990;82(5):1595-606.

4. Bruder O, Wagner A, Lombardi M, Schwitter J, Van Rossum A, Pilz G, et al. European cardiovascular magnetic resonance (EuroCMR) registry-multi national results from 57 centers in 15 countries. J Cardiovasc Magn Reson. 2013;15(1):9.

5. Kramer CM, Barkhausen J, Bucciarelli-Ducci C, Flamm SD, Kim RJ, Nagel E. Standardized cardiovascular magnetic resonance imaging (CMR) protocols: 2020 update. J Cardiovasc Magn Reson. 2020;22(1):17.
6. Mishra RK, Dorbala S, Logsetty G, Hassan A, Heinonen T, Schelbert HR, et al. Quantitative relation between hemodynamic changes during intravenous adenosine infusion and the magnitude of coronary hyperemia implications for myocardial perfusion imaging. J Am Coll Cardiol. 2005;45:553-8.

7. Modi BN, Rahman H, Sherif SA, Ellis H, Kseniia E, Chiribiri A, et al. Is heart rate response a reliable marker of adenosine-induced coronary hyperemia? Int J Cardiovasc Imaging. 2018:34:1117-25.

8. Gulsin GS, Abdelaty AMK, Shetye A, Lai FY, Bajaj A, Das I, et al. Haemodynamic effects of pharmacologic stress with adenosine in patients with left ventricular systolic dysfunction. Int J Cardiol. 2019;278:157-61.

9. Bravo PE, Hage FG, Woodham RM, Heo J, Iskandrian AE. Heart rate response to adenosine in patients with diabetes mellitus and normal myocardial perfusion imaging. Am J Cardiol. 2008;102:1103-6.

10. Hage FG, Heo J, Franks B, Belardinelli L, Blackburn B, Wang W, et al. Differences in heart rate response to adenosine and regadenoson in patients with and without diabetes mellitus. Am Heart J. 2009;157(4):771-6.

11. Kellman P, Hansen MS, Nielles-Vallespin S, Nickander J, Themudo R, Ugander $\mathrm{M}$, et al. Myocardial perfusion cardiovascular magnetic resonance: optimized dual sequence and reconstruction for quantification. J Cardiovasc Magn Reson. 2017;19(1):43.

12. Brown LAE, Onciul SC, Broadbent DA, Johnson K, Fent GJ, Foley JRJ, et al. Fully automated, inline quantification of myocardial blood flow with cardiovascular magnetic resonance: repeatability of measurements in healthy subjects. J Cardiovasc Magn Reson. 2018;20(1):48.

13. Xue H, Brown LAE, Nielles-Vallespin S, Plein S, Kellman P. Automatic in-line quantitative myocardial perfusion mapping: processing algorithm and implementation. Magn Reson Med. 2020;83(2):712-30.

14. Manisty C, Ripley DP, Herrey AS, Captur G, Wong TC, Petersen SE, et al. Splenic switch-off: a tool to assess stress adequacy in adenosine perfusion cardiac MR imaging. Radiology. 2015;276(3):732-40.

15. Hosking A, Koulouroudias M, Zemrak F, Moon JC, Rossi A, Lee A, Barnes MR, Boubertakh R, Pugliese F, Manisty C, Petersen SE. Evaluation of splenic switch off in a tertiary imaging centre: validation and assessment of utility. Eur Heart J Cardiovasc Imaging. 2017;18(11):1216-21.

16. Cerqueira MD, Weissman NJ, Dilsizian V, Jacobs AK, Kaul S, Laskey WK, et al. Standardized myocardial segmentation and nomenclature for tomographic imaging of the heart. A statement for healthcare professionals from the Cardiac Imaging Committee of the Council on Clinical Cardiology of the American Heart Association. Circulation. 2002;105(4):539-42.

17. Treuth MG, Reyes GA, He ZX, Cwajg E, Mahmarian JJ, Verani MS. Tolerance and diagnostic accuracy of an abbreviated adenosine infusion for myocardial scintigraphy: a randomized, prospective study. J Nucl Cardiol. 2001;8(5):548-54

18. Tomiyama T, Ichiro KS, Ishihara K, Suda M, Sakurai M, Hakozaki K, et al. Patients with reduced heart rate response to adenosine infusion have low myocardial flow reserve in 13N-ammonia PET studies. Int J Cardiovasc Imaging. 2015;31(5):1089-95.

19. Verdoia M, Erbetta R, Sagazio E, Barbieri L, Negro F, Suryapranata H, et al. Impact of increasing dose of intracoronary adenosine on peak hyperemia duration during fractional flow reserve assessment. Int J Cardiol. 2019;184:16-21.

20. Röther J, Achenbach S, Tröbs M, Blachutzik F, Nef H, Marwan M, et al. Comparison of standard- and high-dose intracoronary adenosine for the measurement of coronary fractional flow reserve (FFR). Clin Res Cardiol. 2016;105(12):1003-10.

21. Sparv D, Götberg M, Harnek J, Persson T, Hardig BM, Erlinge D. Assessment of increasing intravenous adenosine dose in fractional flow reserve. BMC Cardiovasc Disord. 2017;17:60.

22. Karamitsos TD, Ntusi NA, Francis JM, Holloway CJ, Myerson SG, Neubauer S. Feasibility and safety of high-dose adenosine perfusion cardiovascular magnetic resonance. J Cardiovasc Magn Reson. 2010;12(1):66.

23. Asakura M, Asanuma H, Kim J, Liao Y, Nakamaru K, Fujita M, et al. Impact of adenosine receptor signaling and metabolism on pathophysiology in patients with chronic heart failure. Hypertens Res. 2007:30(9):781-7.

24. Headrick JP, Peart JN, Reichelt ME, Haseler $\sqcup$. Adenosine and its receptors in the heart: regulation, retaliation and adaptation . BBA Biomembr. 2011;1808:1413-28

25. Johnston DL, Hodge DO, Hopfenspirger MR, Gibbons RJ. Clinical determinants of hemodynamic and symptomatic responses in 2,000 patients during adenosine scintigraphy. Mayo Clin Proc. 1998;73(4):314-20. 
26. Oudkerk M, Kuijpers D, Prakken NH, Vliegenthart R, van Dijkman PRM, van der Harst Bronovonl P, et al. Caffeine intake inverts the effect of adenosine on myocardial perfusion during stress as measured by T1 mapping. Int J Cardiovasc Imaging. 2016;32:1545-53.

27. Banko LT, Haq SA, Rainaldi DA, Klem I, Siegler J, Fogel J, et al. Incidence of caffeine in serum of patients undergoing dipyridamole myocardial perfusion stress test by an intensive versus routine caffeine history screening. AJC. 2010;105:1474-9.

\section{Publisher's Note}

Springer Nature remains neutral with regard to jurisdictional claims in published maps and institutional affiliations.
Ready to submit your research? Choose BMC and benefit from:

- fast, convenient online submission

- thorough peer review by experienced researchers in your field

- rapid publication on acceptance

- support for research data, including large and complex data types

- gold Open Access which fosters wider collaboration and increased citations

- maximum visibility for your research: over $100 \mathrm{M}$ website views per year

At BMC, research is always in progress.

Learn more biomedcentral.com/submissions 